\title{
Fortalecimiento de la investigación en salud pública para la mejora de la salud
}

J Selva Andina Res Soc. 2012; 3(1):1-2.

La investigación en salud pública constituye un abanico que incluye desde la investigación fundamental hasta la investigación de la práctica clínica, incorporando novedosos avances, evaluación de resultados y difusión de los mismos. En realidad, la investigación en salud pública es considerada multidisciplinar al integrar numerosos factores en su desarrollo. Estableciendo como pilar principal el método científico se profundiza en la investigación básica, clínico-epidemiológica y en servicios de salud. La premisa de calidad y relevancia se plasma con la investigación en el ámbito científico internacional, así como en el quehacer cotidiano y las buenas prácticas biomédicas que deben ser incluidas en la investigación como una tarea habitual. Por lo tanto, la investigación requiere adoptar una posición proactiva de indagación, integrando una inquietud planificada y de fomento continuo del conocimiento. Para ello es preciso que la coordinación internacional sea mejorada, buscando un equilibrio entre la investigación básica y aplicada, así como entre la ciencia y la tecnología. De esta forma la investigación no puede considerarse sin innovación, contemplando como fin último responder a las necesidades de las personas y la sociedad.

La actuación en el conocimiento de los procesos de producción científica requiere de la mayor exhaustividad en los procedimientos y en la expresión eficaz de los resultados. Se constata como imprescindible el establecimiento de principios de revisión explícitos y de evaluación de la adecuación de las acciones, siempre dentro de los estándares de conducta científica y equidad del proceso investigador. En las líneas científicas biomédicas se han de considerar las valoraciones generales que se produzcan relacionadas con el impacto y la calidad de la investigación en salud, dirigiendo los esfuerzos mayoritariamente a las áreas que requieran mayor atención. No obstante, no conviene obviar otras áreas temáticas que pudieran resultar deficitarias o con incidencia menor en la población.

La investigación en salud como fuente de nuevas aplicaciones y desarrollo permite el conocimiento, mejorando el bienestar. Sin embargo, no es comprensible sin la consideración de las necesidades y demandas sociales. Por lo tanto, en la investigación en salud pública, y con objeto de mejorar la salud de la población, es preciso refinar y optimizar los métodos de prevención y profilaxis. El abordaje multidisciplinar y multicéntrico en la investigación aportará una mejor comprensión de los procesos así como soluciones de calidad. La puesta en marcha de estrategias que estimulen el fomento de la investigación redundará en el establecimiento de líneas de actuación conjunta, permitiendo un abordaje general en la potenciación de la investigación biomédica. En este sentido y para la mejora social, cabe destacar como 
especialmente relevante la sensibilización de los investigadores en la incentivación de la detección de problemas sociales.

Según lo comentado se estima la necesidad de establecer un marco adecuado para la investigación en salud pública en países deficitarios, con resultados que repercutan en el avance del bienestar de las personas, alentando a las administraciones sanitarias y gobiernos correspondientes a la adopción de medidas oportunas. Por lo tanto, el fin primordial ha de ser la protección y mejora de la salud de las personas. Esta finalidad específica se posiciona en la frontera entre la investigación fundamental y el desarrollo, por lo que el aporte de ideas desde la práctica clínica deberá utilizarse en el tratamiento de problemas de salud y en el avance de la prevención. Al mismo tiempo, la promoción de hábitos formativos en salud pública contribuirá a una mejor transferencia de conocimiento y la implantación de costumbres saludables que colaboren hacia el desarrollo. Se presenta una extraordinaria oportunidad para la instauración de la investigación en salud pública, a través de la consideración prioritaria de los principales problemas de salud y la aportación de soluciones viables que contribuyan a mejorar la situación existente. La superación de los retos en salud dirigirá sin duda a una mejora de la sostenibilidad en el siglo XXI, produciendo un beneficio social, favoreciendo el progreso de la humanidad en procesos tecnológicos, comunicativos y de equidad. La competitividad entre grupos de investigación debe entenderse como un mecanismo de enfoque constructivo, con la finalidad última de mejora de la sociedad. A su vez, esta última deberá entender y valorar los avances producidos a través de la investigación biomédica, por lo que se deberá hacer un esfuerzo de comunicación y difusión científica, garantizando la fiabilidad científica de la investigación. En especial se atenderá a un mejor entendimiento de los resultados de investigación, principalmente aquellos cuya valoración objetiva de los beneficios sociales no sea inmediata o resulte compleja. En el proceso de investigación en salud pública se buscará con rigor y ética la mayor eficacia posible (bien entendida), con objeto de prevenir enfermedades, proteger y promocionar la salud, y mejorar tecnológicamente entre otros; persiguiendo como fin último alcanzar una mejor calidad de vida en la población.

Enrique Gea-Izquierdo Universidad de Málaga. Cátedra de Seguridad y Salud en el Trabajo-Málaga. España. Dirección de contacto. Tel.: + 34677454317. E-mail: enriquegea@telefonica.net 